\title{
Effective charge of colloidal particles
}

Cite as: J. Chem. Phys. 121, 12100 (2004); https://doi.org/10.1063/1.1824013

Submitted: 23 August 2004 . Accepted: 01 October 2004 . Published Online: 30 November 2004

Alexandre Diehl, and Yan Levin

\section{ARTICLES YOU MAY BE INTERESTED IN}

Charge renormalization, osmotic pressure, and bulk modulus of colloidal crystals: Theory

The Journal of Chemical Physics 80, 5776 (1984); https://doi.org/10.1063/1.446600

Effective charge saturation in colloidal suspensions

The Journal of Chemical Physics 117, 8138 (2002); https://doi.org/10.1063/1.1511507

Limiting Laws and Counterion Condensation in Polyelectrolyte Solutions I. Colligative Properties

The Journal of Chemical Physics 51, 924 (1969); https://doi.org/10.1063/1.1672157

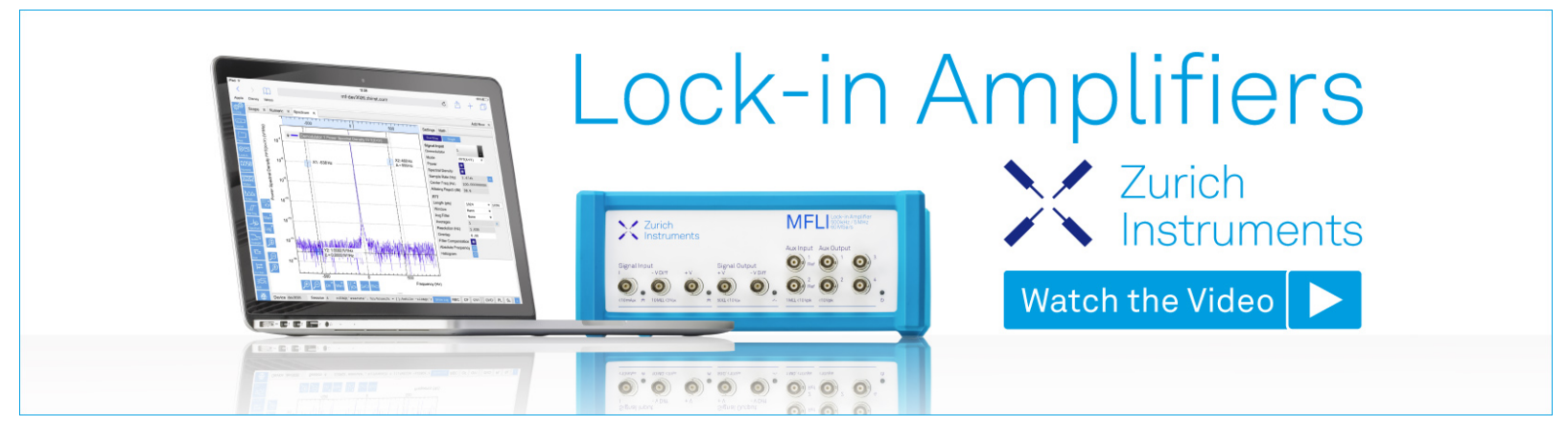

J. Chem. Phys. 121, 12100 (2004); https://doi.org/10.1063/1.1824013 


\title{
Effective charge of colloidal particles
}

\author{
Alexandre Diehla) \\ Departamento de Física, Universidade Federal do Ceará, Caixa Postal 6030, CEP 60455-760, \\ Fortaleza, CE, Brazil \\ Yan Levin ${ }^{\text {b) }}$ \\ Instituto de Física, Universidade Federal do Rio Grande do Sul Caixa Postal 15051, CEP 91501-970, \\ Porto Alegre, RS, Brazil
}

(Received 23 August 2004; accepted 1 October 2004)

\begin{abstract}
A new dynamical definition of the effective colloidal charge, especially suitable for the Monte Carlo and Molecular-dynamics simulations, is proposed. It is shown that for aqueous colloidal suspensions containing monovalent counterions the "dynamical" effective charge agrees perfectly with the "statistical" effective charge calculated using the Alexander prescription. In the case of multivalent ions, the effective charge behaves in a qualitatively different way from the predictions of the Poisson-Boltzmann theory. (C) 2004 American Institute of Physics. [DOI: 10.1063/1.1824013]
\end{abstract}

\section{INTRODUCTION}

While the bare charge of a colloidal particle can be easily measured by chemical methods such as titration, its effective charge is much more illusive. In the absence of a specific adsorption, the colloidal charge renormalization arises from strong electrostatic coupling between the ionized surface groups and the counterions. A number of dissociated counterions can re-condense onto the colloidal particle forming a colloid-counterion complex. These complexes behave as new entities, strongly affecting all the thermodynamic properties of the colloidal suspension.

The phenomenon of counterion condensation has been known in the polyelectrolyte literature for close to four decades under the name of the "Manning condensation." 1,2 A quantitative analysis of the charge renormalization in the colloidal lattices is comparatively more recent, initiated by the pioneering work of Alexander et al. in the early 1980th. ${ }^{3}$

A major stumbling block to the development of a quantitative theory of colloidal charge renormalization is the absence of an explicit experimental or simulational method to directly probe the effective colloidal charge. Since the experiments yield quantities other than the effective charge, the experimental data must be converted into the effective charges through the use of some theory. ${ }^{4}$ For example, the Smoluchowski equation relates the electrophoretic mobility to the zeta potential at the shear plane of the colloidal particle. While it is quite reasonable to argue that the Smoluchowski equation should be quite accurate for thin double layers, one must still locate the shear plane, and then use a different theory to translate the zeta potential into the effective charge. ${ }^{5}$ Clearly, reliance on a number of different theories in order to extract the effective charge, makes it very difficult to judge the accuracy of the final result.

Similarly, up to now the Monte Carlo and moleculardynamics simulations provided only an indirect route to the

\footnotetext{
${ }^{a)}$ Electronic address: diehl@fisica.ufc.br

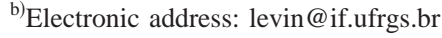

effective colloidal charge. ${ }^{6-8}$ While from the simulations it is relatively straightforward to obtain the thermodynamic functions, ${ }^{9}$ it is much more tricky to translate these into the effective charges. For example, the osmotic pressure is a natural barometer of the degree of counterion condensation. ${ }^{10}$ Unfortunately, it is in general quite difficult to write an expression for the osmotic pressure in terms of the renormalized charge. ${ }^{11-17}$ One must then rely on some subjective measure of the effective charge based on the colloid-counterion correlation functions. ${ }^{18-20}$

It is the goal of this paper to begin to rectify this situation. Specifically, for salt free suspensions, we shall propose a general criterion which allows a straightforward calculation of the effective charge using either the molecular dynamics or the Monte Carlo simulations.

\section{COUNTERION ASSOCIATION AND SIMULATION METHODOLOGY}

\section{A. The model}

We shall work in the context of the Wigner-Seitz cell model (WS) of a colloidal lattice. A colloidal particle of radius $a$ and charge $Z$ is located at the center of a spherical cell of radius $R$. To preserve the overall charge neutrality, the cell also contains counterions of radius $a_{c}=2 \AA$. The solvent is treated as a uniform continuum of a dielectric constant $\epsilon$. In this paper we shall explore two distinct Cases: (1) The cell contains only monovalent counterions and (2) the cell contains multivalent counterions. In conclusions, we will discuss a more general Case (3) of the the cell containing both counterions and coions.

To start the discussion of colloidal charge renormalization we first consider the Case (1). Our objective is to come up with a general criterion which would allow us to clearly define when the counterions are associated (condensed) with the colloid. Mechanically, association is easily seen from the dynamics of particle trajectories. If, in the absence of external fields, the particle trajectories are confined to a finite volume (in the center of mass coordinate system), we can say 
that the particles belong to a bound state, or are "associated." For a two body system, the criterion for association is particularly simple. It is sufficient that the total energy of the system (kinetic plus potential) be negative. Unfortunately, this criterion already breaks down for a three body problem. Thus, if we consider a classical Helium atom-two electrons (of charge $-q$ ) orbiting a nucleus of charge $+2 q$-and start with a initial condition such that the total energy $E$ is negative, in general this does not guarantee that the atom will not ionize in a fairly short time. ${ }^{21,22}$ On the other hand, statistically there seems to be a fairly clear distinction between the condensed and the free counterions. In the case of an aqueous colloidal suspension with monovalent counterions there is a "natural" way of defining the effective colloidal charge by asymptotically matching the exact electrostatic potential to the solution of the linearized Poisson-Boltzmann (PB) equation, this is known as the Alexander prescription. ${ }^{3,23,24}$

The Alexander prescription is particularly straight forward to use for aqueous suspensions with monovalent counterions, since in this case the correlations between the counterions are negligible and the Poisson-Boltzmann WS cell theory is very accurate. ${ }^{25,26}$ The asymptotic match of the numerical solution of the nonlinear PB equation with the linearized equation, then determines the effective colloidal charge. The Alexandre prescription, however, is difficult to implement directly within the Monte Carlo or moleculardynamics simulations since, in practice, there is never sufficient accuracy to do a reliable match. Furthermore, since for multivalent counterions (or low dielectric solvents) the ionion correlations become important, the PB equation breaks down and up to now there was no reliable prescription for obtaining the effective colloidal charge.

The fact that statistically the effective charge is a well defined quantity, suggest that in the limit of large number of counterions there should also exist a dynamical prescription for charge renormalization. The simplest such criterion might be to suppose a linear relationship between the kinetic and the electrostatic potential energies of the condensed counterions. We shall, therefore, assume a counterion to be associated with the colloid if

$$
U \leqslant-\chi K,
$$

where $K=p^{2} / 2 m$ is the kinetic energy of the counterion, $U$ is its electrostatic energy, and $\chi$ is a proportionality factor. At this point, Eq. (1) is only a proposition. Whether it correctly describes the counterions condensation will depend on its ability to reproduce the charge renormalization obtained using the statistical Alexander prescription.

\section{B. Simulation method}

The implementation of Eq. (1) within the moleculardynamics simulation is quite straight forward and will not be addressed in this article. Here we shall restrict our attention to the Monte Carlo (MC) method. The simulation procedure adopted in this case is the following. A MC simulation is run during $1-5 \times 10^{7} \mathrm{MC}$ steps until the system is equilibrated. To each ion of the equilibrium configuration is then assigned a momentum vector $\boldsymbol{p}=\left(p_{x}, p_{y}, p_{z}\right)$, with the component $p_{i}$ drawn from the normalized Boltzmann distribution

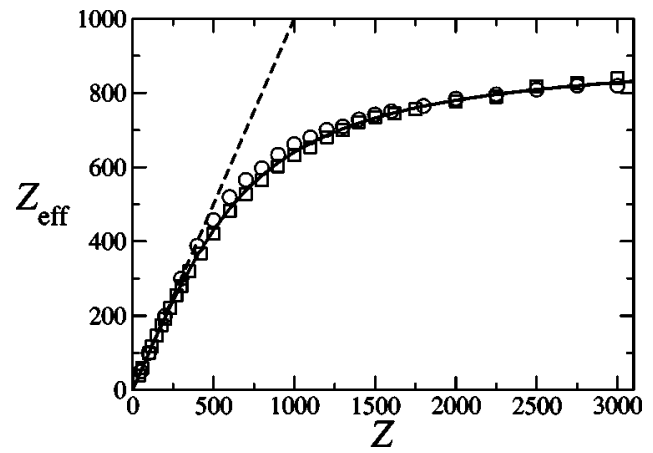

FIG. 1. The effective charge of colloidal particles of radius $a=720 \AA$ inside a WS cell of $R=2880 \AA$, as a function of the bare charge. Symbols are the results of the MC simulation: Open squares for Eq. (1) with $\chi=4 / 3$ and open circles for criterion (3). The solid curve is obtained using the Alexandre criterion within the Poisson-Boltzmann theory. The dashed line $Z_{\text {eff }}$ $=Z$, shows that there is no charge renormalization for weakly charged colloids. The effective charge saturates in the limit $Z \rightarrow \infty$.

$$
P\left(p_{i}\right)=\frac{e^{-p_{i}^{2} / 2 m k_{B} T}}{\sqrt{2 \pi m k_{B} T}},
$$

where $k_{B}$ is the Boltzmann constant and $T$ is the temperature. The number associated counterions $n^{\star}$ in this configuration is then determined using the criterion (1). The simulation is continued for another $1-5 \times 10^{6} \mathrm{MC}$ steps to decorrelate the configurations and the number of condensed counterions is calculated again. The average number of condensed counterions $\bar{n}^{\star}$ is calculated using the block average with 10 uncorrelated configurations. The effective colloidal charge is then $Z_{\text {eff }}=Z-\bar{n}^{\star}$. To speed up the simulation a $1 / r$ biased sampling was used in addition to the usual Metropolis algorithm. $^{27}$

\section{RESULTS AND DISCUSSION}

We shall start our discussion with the Case (1). For dilute aqueous suspension (large $R$ ) with small counterions we find that Eq. (1) with $\chi=4 / 3$ produces the effective colloidal charges almost identical to the ones obtained using the Alexander prescription within the PB theory, see Fig. 1. It is important to stress that under these conditions the PB equation is extremely accurate, and the effective charges obtained on its basis can, therefore, be taken as "exact" within the WS cell formalism. It is quite amazing that two such apparently different criterions lead to almost identical results. Furthermore, we find that a modification of the criterion (1) which replaces the instantaneous kinetic energy of a particle by the average kinetic energy $\langle K\rangle=3 k_{B} T / 2$ affects very little the value of the renormalized charge, see Fig. 1. Since this modification significantly speeds up the simulations, it will be adopted in the future discussion. The counterion is, then, said to be associated if

$$
U \leqslant-2 k_{B} T \text {. }
$$

Clearly, the criterion (3) is of practical relevance only if it is universal, i.e., independent of other parameters of the colloidal suspension. We can test that this is the case by looking at different colloidal volume fractions. Indeed we find an excellent agreement between the effective charges 


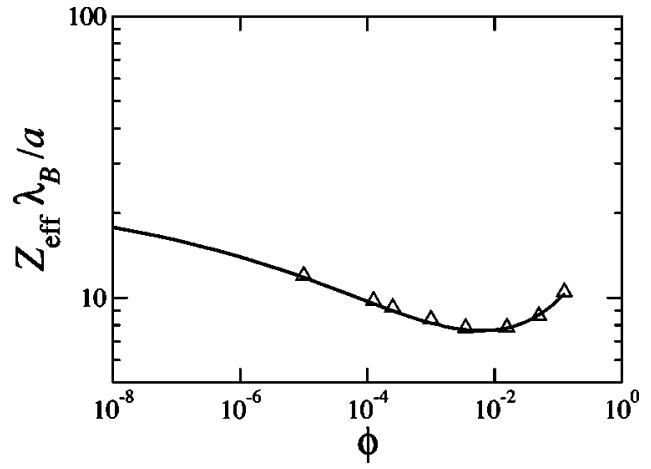

FIG. 2. Variation of the effective charge with the colloidal volume fraction $Z=2000$. The symbols are the result of the MC simulation with the association criterion given by Eq. (3). The solid curve is obtained using the Alexander prescription within the PB theory. The colloidal particle radius is $a=720 \AA$.

calculated using the Alexander prescription within the PB theory and those obtained on the basis of Eq. (3). As might be expected the disagreement appears only for large colloidal volume fractions, when the correlational effects due to ion hard core repulsion become important and the PB equation fails. In Fig. 2, we compare the saturation value of the effective charge as a function of colloidal volume fraction, obtained using the MC simulation and the criterion (3), with the saturation value of the effective charge determined using the Alexander prescription within the PB theory. We see a perfect agreement between the dynamical and the Alexander criterion, up to volume fractions as large as $15 \%$.

Case (2). Convinced of the general validity of the dynamical criterion (3) we can now apply it to the situations in which the Poisson-Boltzmann theory fails and the Alexander criterion is difficult to implement directly. Consider a colloidal particle inside a WS cell in the presence of $n=Z / \alpha$ $\alpha$-valent counterions. Increase of the counterion valence leads to stronger colloid-counterion coupling, which should result in a larger charge renormalization. The effective charge is now $Z_{\text {eff }}=Z-\alpha n^{\star}$, where $n^{\star}$ is the number of associated counterions. To simplify the notation we have dropped the bar over $n^{\star}$.

We first consider the charge renormalization of relatively large colloidal particles of radius $a=720 \AA$. In Fig. 3 the

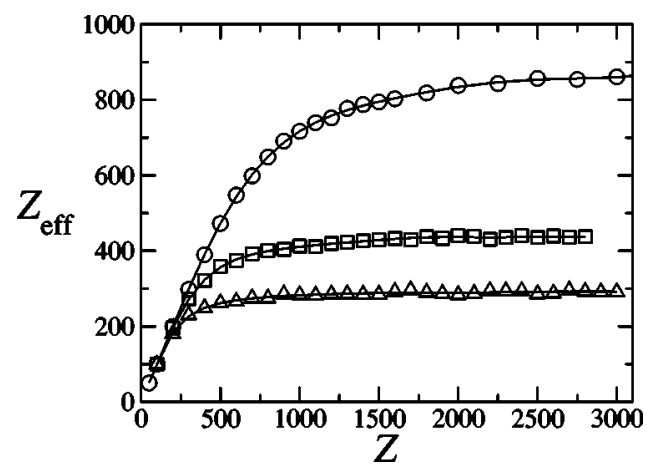

FIG. 3. The effective charge of colloidal particles of radius $a=720 \AA$ inside a WS cell of $R=7200 \AA$, with counterions of either, $\alpha=1$ (open circles), 2 (open squares), or 3 (open triangles). The effective charge, as a function of the bare charge, appears to saturate in all three cases.

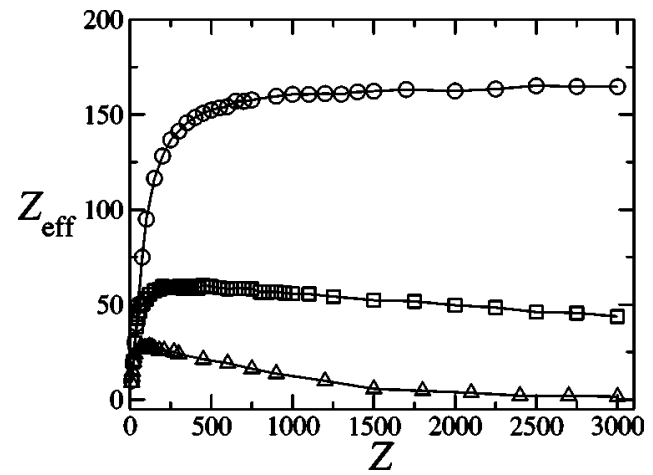

FIG. 4. The effective charges of colloidal particles of radius $a=100 \AA$ inside a WS cell of $R=7200 \AA$, with counterions of different valences. For these particles, in the presence of divalent or trivalent counterions the effective charge, as a function of bare charge, reaches maximum and then decreases. The symbols are the same as in Fig. 3.

effective charge is plotted as a function of the bare charge for three different $\alpha$ 's. It is evident that while the saturation value of the effective charge decreases with the increase of the counterion valence, all three curves have a very similar shape. Next, we consider a smaller colloidal particle of radius $a=100 \AA$ A , Fig. 4. In this case a qualitatively different behavior is obtained with the multivalent, as compared to the monovalent counterions. ${ }^{28}$ While for monovalent counterions the effective charge, as a function of the bare charge, still saturates in the limit $Z \rightarrow \infty$, for the multivalent counterions the effective charge reaches maximum and then decreases going to zero in the limit $Z \rightarrow \infty$. The different behavior of the effective charge in Figs. 3 and 4 is the result of electrostatic correlations between the counterions. The strength of ionic correlations can be quantified by the plasma parameter $^{29}$

$$
\Gamma=\frac{\alpha^{2} q^{2}}{\epsilon d k_{B} T}=\frac{\alpha^{2} \lambda_{B}}{d}
$$

where $d$ is the characteristic distance between the condensed counterions and $\lambda_{B} \approx 7.2 \AA$, for water. If the condensed counterions are located in a thin layer near the colloidal surface, the averaged inter-ionic distance $d$ satisfies $n \pi(d / 2)^{2}$ $=4 \pi a^{2}$, so that $d \approx 4 a / \sqrt{n^{\star}}$. This approximation should be fairly good for multivalent counterions which are tightly bound to the colloid, it will, however, strongly underestimate the separation between the associated monovalent counterions. For strongly charged colloids, the number of condensed counterions is $n^{\star} \approx Z / \alpha$. Substituting $d$ into Eq. (4) we find

$$
\Gamma=\frac{\alpha^{3 / 2} \lambda_{B} \sqrt{Z}}{4 a} .
$$

The strong counterion correlations limit corresponds to $\Gamma$ $>1$. For colloidal particles of radius $a=720 \AA$, with divalent counterions, the correlations become important only for $Z>20000$. For trivalent counterions this value drops down to 6000 , which is still too large to be seen on the scale of Fig. 3. On the other hand, for colloids with $a=100 \AA$ and divalent counterions, the electrostatic correlations are relevant already for $Z>380$, while for trivalent counterions the 
threshold drops down to $Z=115$. These values are consistent with the location of the maximums in $Z_{\text {eff }}$ appearing in Fig. 4.

\section{CONCLUSIONS}

We have presented a new method for calculating the effective charge of colloidal particles. The method is particularly applicable to Monte Carlo and molecular-dynamics simulations. For monovalent counterions the new condensation criterion results in effective charges identical to the ones obtained using the traditional Alexander prescription. The dynamical condensation criterion, however, is also applicable in the situations in which the Alexander prescription is very difficult to implement directly, i.e., strong electrostatic correlations between the counterions. The theoretical challenge now is to understand the dynamical basis for the condensation criterion given by Eq. (1).

A possible application of the present work may be to the study of the charge reversal in colloidal suspension containing multivalent ions and salt. The WS cell, in this case, contains monovalent counterions, $\alpha$-valent counterions, and monovalent coions. The effective colloidal charge is then $Z_{\text {eff }}=Z-\alpha n_{\alpha}^{\star}-n_{+}^{\star}+n_{-}^{\star}$, where $n_{\alpha}^{\star}$ is the number of condensed multivalent counterions, $n_{+}^{\star}$ is the number of condensed monovalent counterions, and $n_{-}^{\star}$ is the number of condensed coions. Because of the many-body nature of the electrostatic interactions, coion condensation must be taken into account in the definition of the effective colloidal charge.

It is important to also keep in mind that in suspensions containing both counterions and coions, besides association with colloid, the inter-ionic association between counterions and coions is also possible. ${ }^{30,31}$ As formulated above, the condensation criterion Eq. (3) will not distinguish between these two effects. In the charge symmetric case (only monovalent counterions and coions present) the cluster formation should not affect the renormalized colloidal charge, since the average charge of clusters is zero. However, in the charge asymmetric case cluster formation between the counterions and coions will invalidate a straightforward application of the association criterion (3). This will be the subject of the future work.

In the present study we have used a simplified model of a colloidal suspension in which both colloids and microions are treated as hard spheres with point charge at the center and the solvent is a dielectric continuum. While this model is sufficient to study the electrostatic properties of rigid colloidal particles, ${ }^{32}$ it will certainly fail as soon as the length scales on the order of few angstroms become important. This is particularly the case when one deals with the structural properties of biomolecules. ${ }^{33-35}$ Under these conditions reliance on simple models may be quite misleading. ${ }^{36,37}$

\section{ACKNOWLEDGMENTS}

This work was supported in part by the Brazilian agencies CNPq, Capes and Fapergs.

${ }^{1}$ G. S. Manning, J. Chem. Phys. 51, 924 (1969).

${ }^{2}$ G. S. Manning, Q. Rev. Biophys. 2, 179 (1978).

${ }^{3}$ S. Alexander, P. M. Chaikin, P. Grant, G. J. Morales, P. Pincus, and D. Hone, J. Chem. Phys. 80, 5776 (1984).

${ }^{4}$ P. Wette, H. J. Schope, and T. Palberg, J. Chem. Phys. 116, 10981 (2002).

${ }^{5}$ W. B. Russel, D. A. Saville, and W. R. Schowalter, Colloidal Dispersions (Cambridge University Press, Cambridge, 1989).

${ }^{6}$ M. Deserno, F. Jimenez-Angeles, C. Holm, and M. Lozada-Cassou, J. Phys. Chem. 105, 10983 (2001).

${ }^{7}$ M. Tanaka and A. Y. Grosberg, J. Chem. Phys. 115, 567 (2001).

${ }^{8}$ M. Tanaka, Phys. Rev. E 68, 061501 (2003).

${ }^{9}$ H. Wennerströn, B. Jönsson, and P. Linse, J. Chem. Phys. 76, 4665 (1982).

${ }^{10}$ M. J. Stevens, M. L. Falk, and M. O. Robbins, J. Chem. Phys. 104, 5209 (1995)

${ }^{11}$ Y. Levin, M. C. Barbosa, and M. N. Tamashiro, Europhys. Lett. 41, 123 (1998).

${ }^{12}$ P. B. Warren, J. Chem. Phys. 112, 4683 (2000).

${ }^{13}$ A. Diehl, M. C. Barbosa, and Y. Levin, Europhys. Lett. 53, 86 (2001).

${ }^{14}$ M. Deserno and H. H. Grünberg, Phys. Rev. E 66, 011401 (2002).

${ }^{15}$ J. F. Dufreche, T. O. White, and J. P. Hansen, Mol. Phys. 101, 1741 (2003).

${ }^{16}$ M. N. Tamashiro and H. Schiessel, J. Chem. Phys. 119, 1855 (2003).

${ }^{17}$ E. Trizac and Y. Levin, Phys. Rev. E 69, 031403 (2004).

${ }^{18}$ L. Belloni, J. Chem. Phys. 85, 519 (1986).

${ }^{19}$ L. Belloni, Colloids Surf., A 140, 227 (1998).

${ }^{20}$ J. P. Hansen and H. Löwen, Annu. Rev. Phys. Chem. 51, 209 (2000).

${ }^{21}$ T. Yamamoto and K. Kaneko, Phys. Rev. Lett. 70, 1928 (1993).

${ }^{22}$ J. De Luca, Phys. Rev. E 62, 2060 (2000).

${ }^{23}$ E. Trizac, L. Bocquet, and M. Aubouy, Phys. Rev. Lett. 89, 248301 (2002).

${ }^{24}$ L. Bocquet, E. Trizac, and M. Aubouy, J. Chem. Phys. 117, 8138 (2002).

${ }^{25}$ M. Deserno, C. Holm, and S. May, Macromolecules 33, 199 (2000).

${ }^{26}$ Y. Levin, E. Trizac, and L. Bocquet, J. Phys.: Condens. Matter 15, S3523 (2003).

${ }^{27}$ D. Frenkel, Understanding Molecular Simulation (Academic, New York, 1996).

${ }^{28}$ R. D. Groot, J. Chem. Phys. 95, 9191 (1991).

${ }^{29}$ Y. Levin, Rep. Prog. Phys. 65, 1577 (2002).

${ }^{30}$ N. Bjerrum, Kgl. Dan. Vidensk. Selsk. Mat.-fys. Medd. 7, 1 (1926).

${ }^{31}$ Y. Levin and M. E. Fisher, Physica A 225, 164 (1996).

${ }^{32}$ M. N. Tamashiro, Y. Levin, and M. C. Barbosa, Eur. Phys. J. B 1, 337 (1998).

${ }^{33}$ M. K. Gilson et al., J. Mol. Biol. 184, 503 (1985).

${ }^{34}$ R. Penfold, J. Warwicker, and B. Jonsson, J. Phys. Chem. B 102, 8599 (1998).

${ }^{35}$ V. Spassov and D. Bashford, Protein Sci. 7, 554 (1998).

${ }^{36}$ P. Auffinger, S. LouiseMay, and E. Westhof, Faraday Discuss. 103, 151 (1996).

${ }^{37}$ P. Auffinger and E. Westhof, Curr. Opin. Struct. Biol. 8, 227 (1998). 